\title{
Representaciones sociales de la docencia universitaria y de las prácticas pedagógicas desde la mirada de estudiantes de ciencias de la salud
}

\author{
Social Representations of University Teaching and Pedagogical Practices \\ from the Perspective of Students of Health Sciences
}

Representações sociais do ensino universitário e das práticas pedagógicas na perspectiva de estudantes de ciências da saúde

\author{
Jesús Roberto Garay Núñez \\ Universidad Autónoma de Sinaloa, México \\ jrgarayn@hotmail.com \\ https://orcid.org/0000-0002-0868-1344
}

\section{Resumen}

Estudiar y reflexionar acerca de las representaciones sociales de la docencia universitaria y de las prácticas pedagógicas desde la mirada de los estudiantes de ciencias de la salud permite realizar un análisis de la realidad que se vive en las instituciones públicas de educación superior en México. El objetivo del presente trabajo fue determinar el núcleo figurativo de las representaciones sociales de la docencia universitaria y de las prácticas pedagógicas de profesores del área de la salud. Metodológicamente, se utilizó la técnica de redes semánticas naturales para determinar las representaciones sociales y las prácticas pedagógicas del docente universitario desde la perspectiva de los estudiantes. El valor de las redes semánticas naturales reside en que las taxonomías obtenidas son generadas de manera directa de la memoria semántica del sujeto, y el orden otorgado va de acuerdo con su escala de valores y percepciones. Como parte de los resultados, se determinó que el núcleo figurativo "Docencia universitaria" recae en el concepto central de estar preparado para impartir la clase, seguido 


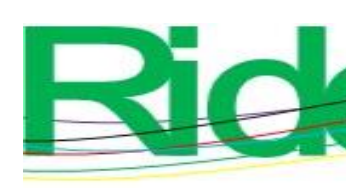

Revista Iberoamericana para la Investigación y el Desarrollo Educativo ISSN $2007-7467$

de los conceptos que integran el sistema periférico de la representaciones sociales: confianza, responsable, comprometido y asertivo. Todos estos componentes son básicos para determinar las redes de asociaciones. En tanto que para el núcleo figurativo "Prácticas pedagógicas", el concepto central es el de aplicación de examen como herramienta principal para evaluar los aprendizajes de los estudiantes del área de la salud, seguido de los conceptos que integran el sistema periférico de las representaciones sociales: exposición, cuadro sinóptico, mapas conceptuales y resumen. Todos estos componentes son básicos para determinar las redes de asociaciones. En conclusión, en cuanto a las redes de asociaciones de las respuestas de los universitarios de ciencias de la salud que se presentan en la configuración del núcleo central de las representaciones sociales de la docencia universitaria, que corresponden a la primera red de asociación, el concepto central alude a la preparación académica de los docentes. La segunda red de asociaciones de las representaciones sociales de las prácticas pedagógicas, por su parte, permite determinar el contenido y organización de la representación social, la cual es determinada por la evaluación de los aprendizajes a través del examen.

Palabras claves: antropología de la educación, colectivismo, psicología social.

\section{Abstract}

Studying and reflecting on the social representations of university teaching and pedagogical practices from the perspective of health science students allows for an analysis of the reality that exists in public institutions of higher education in Mexico. The objective of the present work was to determine the figurative nucleus of the social representations of university teaching and of the pedagogical practices of professors in the health area. Methodologically, the natural semantic networks technique was used to determine the social representations and pedagogical practices of the university teacher from the perspective of the students. The value of natural semantic networks is that the taxonomies obtained are generated directly from the subject's semantic memory, and the order given is in accordance with their scale of values and perceptions. As part of the results, it was determined that the figurative nucleus "University teaching" falls on the central concept of being prepared to teach the class, followed by the concepts that make up the peripheral system of social representations: trust, responsible, committed and assertive. All these components are basic to determine the association networks. While for the figurative nucleus "Pedagogical practices", the central 


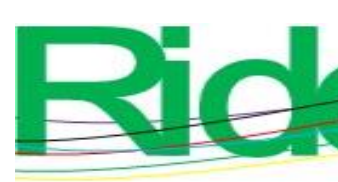

Revista Iberoamericana para la
Investigación y el Desarrollo Educativo
ISSN $2007-7467$

concept is that of exam application as the main tool to evaluate the learning of students in the health area, followed by the concepts that make up the peripheral system of social representations : exhibition, synoptic table, concept maps and summary. All these components are basic to determine the association networks. In conclusion, regarding the networks of associations of the responses of the university students of health sciences that are presented in the configuration of the central nucleus of the social representations of university teaching, which correspond to the first association network, the concept central refers to the academic preparation of teachers. The second network of associations of social representations of pedagogical practices, meanwhile, allows determining the content and organization of social representation, which is determined by the evaluation of learning through the exam.

Key words: anthropology of education, collectivism, social psychology.

\section{Resumo}

Estudar e refletir sobre as representações sociais do ensino universitário e das práticas pedagógicas na perspectiva de estudantes de ciências da saúde permite analisar a realidade existente em instituições públicas de ensino superior no México. O objetivo do presente trabalho foi determinar o núcleo figurativo das representações sociais do ensino universitário e das práticas pedagógicas dos professores da área da saúde. Metodologicamente, a técnica de redes semânticas naturais foi utilizada para determinar as representações sociais e práticas pedagógicas do professor universitário na perspectiva dos alunos. O valor das redes semânticas naturais é que as taxonomias obtidas são geradas diretamente da memória semântica do sujeito, e a ordem dada está de acordo com sua escala de valores e percepções. Como parte dos resultados, determinou-se que o núcleo figurativo "ensino universitário" recai sobre o conceito central de estar preparado para ministrar a aula, seguido pelos conceitos que compõem o sistema periférico de representações sociais: confiança, responsabilidade, comprometimento e assertividade . Todos esses componentes são básicos para determinar as redes de associação. Já para o núcleo figurativo "Práticas pedagógicas", o conceito central é o da aplicação do exame como principal ferramenta para avaliar a aprendizagem dos alunos da área da saúde, seguido pelos conceitos que compõem o sistema periférico de representações sociais. : exposição, tabela sinótica, mapas conceituais e resumo. Todos esses componentes são básicos para determinar as redes de associação. Concluindo, 


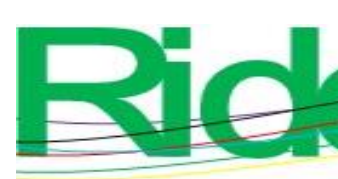

Revista Iberoamericana para la
Investigación y el Desarrollo Educativo
ISSN $2007-7467$

em relação às redes de associações das respostas dos estudantes universitários de ciências da saúde que são apresentadas na configuração do núcleo central das representações sociais do ensino universitário, que correspondem à primeira rede de associações, o conceito central refere-se à preparação acadêmica dos professores. A segunda rede de associações de representações sociais de práticas pedagógicas, por outro lado, permite determinar o conteúdo e a organização da representação social, que é determinada pela avaliação da aprendizagem através do exame.

Palavras-chave: antropologia da educação, coletivismo, psicologia social.

Fecha Recepción: Febrero 2020

Fecha Aceptación: Julio 2020

\section{Introducción}

Estudiar y reflexionar acerca de las representaciones sociales de la docencia universitaria y de las prácticas pedagógicas desde la mirada de los estudiantes universitarios de ciencias de la salud permite realizar un análisis de la realidad que se vive en las instituciones públicas de educación superior de nuestro país. La formación y actualización docente adquieren un papel medular en la calidad de la educación; una y otra deben de ajustarse a los cambios permanentes que se viven en las políticas educativas nacionales e internacionales.

El siglo XXI ha traído consigo una enorme cantidad de transformaciones en muchas esferas de la vida social, educativa, tecnológica, científica y familiar. Estas transformaciones han tocado de manera particular el ámbito educativo, con reformas en sus modelos educacionales; y con ello los estudiantes universitarios han acometido la tarea de romper con paradigmas de corte positivista, en donde el alumno era visto como una fuente restringida por los arreglos contingenciales del profesor programador, los cuales se establecían incluso antes de la situación instruccional (Hernández, 2006).

En el ámbito de la educación superior, de acuerdo con Amber y Suárez (2016), estos cambios principalmente están vinculados a tres procesos: a) masificación de los sistemas de educación superior, que conlleva a la entrada de "nuevos estudiantes" o "estudiantes no tradicionales"; b) creciente internacionalización de la formación, que implica un aumento permanente de la movilidad en el ámbito universitario e introducción de nuevas tecnologías 


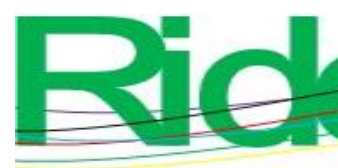

Revista Iberoamericana para la Investigación y el Desarrollo Educativo ISSN 2007 - 7467

de la información y la comunicación (TIC), y c) progresiva incorporación de la perspectiva del aprendizaje a lo largo de la vida como enfoque regulador de la acción educativa.

En este escenario, una demanda generalizada es que la formación profesional se base en una enseñanza de calidad, responsabilidad de todos los actores sociales involucrados, directivos, docentes, estudiantes y administradores, aunque de manera particular sobresale el papel determinante de los docentes en el avance de la calidad de la enseñanza. La importancia de la evaluación de la docencia se deriva del potencial que posee como herramienta para contribuir a la profesionalización de los maestros y, con ello, a la mejora de la formación profesional (Rueda y Luna, 2008).

En el caso de la Universidad Autónoma de Sinaloa (UAS), en sesión ordinaria del H. Consejo Universitario realizada el día 9 de julio de 2007, se aprobó el programa educativo de licenciatura en Enfermería, el cual es el primer programa de ciencias de la salud que se diseña bajo el modelo por competencias profesionales integradas. Es desde este cambio curricular que comienzan a reinventarse las prácticas docentes para un nuevo tipo de profesor y para un nuevo tipo de estudiante. Es decir, la docencia centrada en el aprendizaje, la autorregulación y la emancipación académica.

Aquel sujeto cuyo desempeño y aprendizaje escolar podía ser arreglado desde el exterior se encuentra en un proceso de emancipación y toma de conciencia; ahora participa activamente en su propio aprendizaje. Muchas universidades en México trabajan para dar un giro decisivo y proporcionar autonomía en sus estudiantes mediante modelos como el constructivista (Zúñiga, 2012). Sin embargo, en palabras del propio Zúñiga (2012), esa autonomía no es auténtica: los estudiantes se subsumen al currículo de su institución y generan aprendizajes que se limitan a lo que se les evaluará, y aun así son reificados.

Los fundamentos teórico-pedagógicos del modelo académico de la UAS (2007) sobre una docencia centrada en el aprendizaje se encuentran en lo siguiente:

En las perspectivas de la educación social, el humanismo y el constructivismo, donde se concibe a la persona como ser social responsable y corresponsable en el proceso de aprendizaje. La educación social se enmarca en el proyecto de educación a lo largo de la vida, y apuesta a las nuevas alfabetizaciones (informática, idiomas, técnica), a la inclusión y la igualdad de oportunidades.

Con base en lo anterior, las y los docentes deben asumir la función de organizar, motivar y coordinar lo necesario para alcanzar el propósito de la 


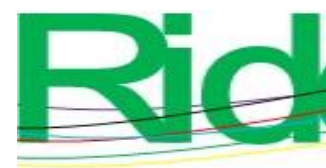

Revista Iberoamericana para la
Investigación y el Desarrollo Educativo
ISSN $2007-7467$

educación integral, con participación de los mismos estudiantes. En esto es necesario que desde la docencia se promueva la toma de conciencia de los estudiantes respecto a sus vínculos con la sociedad, la importancia de conducirse correctamente en ella y aportar para mejorarla y transformarla. Las y los profesores deben contribuir a lograr la armonía, integración, equilibrio y formación de alumnos y alumnas en todos los ámbitos (p. 17).

Ahora bien, ¿cómo es el estudiante del siglo XXI? Esa misma pregunta se hace Tourón (9 de marzo de 2015) y, a modo de respuesta, enlista las siguientes características:

- $\quad$ Se mueven y experimentan.

- $\quad$ El aprendiz es un iniciador.

- Navegan entre diferentes opciones.

- $\quad$ Orientado al proceso y al producto.

- $\quad$ Construye y reconstruye los nuevos significados.

- $\quad$ Está enriquecido por la tecnología multimedia.

- $\quad$ No tiene límites en la gestión de su conocimiento.

- $\quad$ Comparte globalmente.

- $\quad$ Tiende al trabajo colaborativo.

- Soluciona problemas.

- Realiza muchas preguntas.

- $\quad$ Es de sistema abierto.

- Cambia constantemente.

- $\quad$ Considera que no hay un modo correcto de gestionar su aprendizaje.

- Realiza gráficos y otras tareas de forma práctica e inmediata.

Tourón (9 de marzo de 2015) añade al final de esta lista:

Estamos hablando de las necesidades actuales y para un futuro más o menos inmediato que la educación debe abordar para que los aprendices, estudiantes, puedan hacer frente con cierta soltura a las demandas de la construcción social, del desarrollo científico, del mundo laboral. Todas ellas son cambiantes, pero es cierto que si nos centramos en conceptos, en destrezas, en actitudes, en competencias en suma, estaremos promoviendo un bagaje cultural que será más adaptable a lo cambiante (párr. 1). 


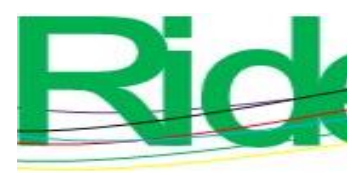

Revista Iberoamericana para la
Investigación y el Desarrollo Educativo
ISSN $2007-7467$

Los estudiantes universitarios tienen una forma particular de percibir o representar al docente universitario y sus prácticas pedagógicas. Este estudio es un acercamiento a esas representaciones que se presentan en la realidad social de las instituciones de educación superior.

Pensar en mejorar la calidad de la educación significa pensar en las prácticas de enseñanza. Las prácticas de enseñanza representan el espacio social en el que se gestan y promueven la motivación por aprender, el compromiso con las exigencias escolares, las estrategias de aprendizaje y la misma enunciación y aceptación de las formas de evaluación; de ahí la importancia de su estudio y perfeccionamiento (Covarrubias, 2011).

Las representaciones sociales constituyen una oportunidad invaluable para acercarnos a la comprensión de los marcos de referencia que sustentan y fundamentan las identidades profesionales y pedagógicas de los profesores, y las formas en que las expresan o proyectan durante el acto educativo. Permiten conocer cómo obtienen, construyen o reconstruyen el propio conocimiento disciplinar y profesional del que participan, y los significados que atribuyen a las prácticas pedagógicas a las que se adscriben (Covarrubias, 2016, p. 89).

Para Jodelet (1986), las representaciones sociales son una manera de interpretar y de pensar nuestra realidad cotidiana, una forma de conocimiento del entramado social. También son la actividad mental desplegada por individuos y grupos a fin de fijar su posición en relación con situaciones, acontecimientos, objetos y comunicaciones que les conciernen

Además, Jodelet (2006, citado en Beltrán y Garay, 2016) señala lo puesto a continuación:

Cuando las personas hacen referencia a los objetos sociales, los clasifican, explican y evalúan, porque tienen una representación social de ese objeto; y un objeto se representa cuando está mediado por una figura; y es solo en esa condición que emerge la representación y el contenido correspondiente. Las personas conocen la realidad que les circunda mediante explicaciones que extraen de los procesos de comunicación y del pensamiento social. Las representaciones sociales sintetizan dichas explicaciones y en consecuencia, hacen referencia a un tipo específico de conocimiento que juega un papel crucial sobre cómo la gente piensa y organiza su vida cotidiana: el conocimiento del sentido común (pp. 6-7). 


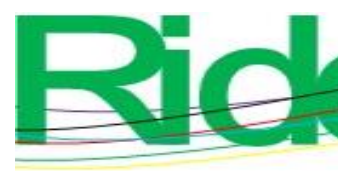

Revista Iberoamericana para la Investigación y el Desarrollo Educativo ISSN $2007-7467$

El sentido común, según Reid (citado en Araya, 2002), es, en principio:

Una forma de percibir, razonar y actuar. El conocimiento del sentido común es conocimiento social porque está socialmente elaborado. Incluye contenidos cognitivos, afectivos y simbólicos que tienen una función no solo en ciertas orientaciones de las conductas de las personas en su vida cotidiana, sino también en las formas de organización y comunicación que poseen tanto en sus relaciones interindividuales como entre los grupos sociales en que se desarrollan.

Las [representaciones sociales], en definitiva, constituyen sistemas cognitivos en los que es posible reconocer la presencia de estereotipos, opiniones, creencias, valores y normas que suelen tener una orientación actitudinal positiva o negativa. Se constituyen, a su vez, como sistemas de códigos, valores, lógicas clasificatorias, principios interpretativos y orientadores de las prácticas, que definen la llamada conciencia colectiva, la cual se rige con fuerza normativa en tanto instituye los límites y las posibilidades de la forma en que las mujeres y los hombres actúan en el mundo (p. 11).

Para Berger y Luckmann (1991, citados en Barbachán, Cajas, Ramos y Sánchez, 2017), la construcción de la realidad social hace referencia a la tendencia fenomenológica de las personas a considerar los procesos subjetivos como realidades objetivas.

Las personas aprehenden la vida cotidiana como una realidad ordenada, es decir, las personas perciben la realidad como independiente de su propia aprehensión, apareciendo ante ellas objetivada y como algo que se les impone.

El mundo de la vida cotidiana es aquel que se da por establecido como realidad. El sentido común que lo constituye se presenta como la "realidad por excelencia", logrando de esta manera imponerse sobre la conciencia de las personas pues se les presenta como una realidad ordenada, objetivada y ontogenizada.

(...) Las inserciones de las personas en diferentes categorías sociales y su adscripción a distintos grupos constituyen fuentes de determinación que inciden con fuerza en la elaboración individual de la realidad social, y esto es, precisamente, lo que genera visiones compartidas de la realidad e 


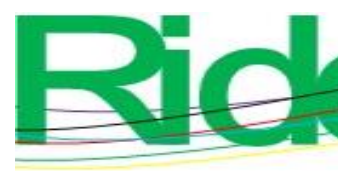

Revista Iberoamericana para la
Investigación y el Desarrollo Educativo
ISSN $2007-7467$

interpretaciones similares de los acontecimientos (Barbachán et al., 2017, pp. 708-709).

La realidad de la vida cotidiana, por tanto, es una construcción intersubjetiva, un mundo compartido. Ello presupone procesos de interacción y comunicación mediante los cuales las personas comparten y experimentan a los otros y a las otras. En esta construcción, la posición social de las personas, así como el lenguaje, juegan un papel decisivo al posibilitar la acumulación o acopio social del conocimiento que se transmite de generación en generación. En resumen, el medio cultural en el que viven las personas, el lugar que ocupan en la estructura social, y las experiencias concretas con las que se enfrentan a diario, influyen en su forma de ser, su identidad social y la forma en que perciben la realidad social. El anterior planteamiento goza de aprobación en un amplio sector de quienes realizan investigación en las ciencias sociales (Ibáñez, 1988).

El objetivo de esta investigación es determinar el núcleo figurativo de las representaciones sociales de la docencia universitaria y de las prácticas pedagógicas de docentes del área de la salud desde la mirada de estudiantes ciencias de la salud de una universidad pública de la ciudad de Culiacán, Sinaloa, México.

\section{Material y métodos}

Se utilizó la técnica de redes semánticas naturales para determinar las representaciones sociales y las prácticas pedagógicas del docente universitario desde la perspectiva de los estudiantes.

El valor de las redes semánticas naturales reside en que las taxonomías obtenidas son generadas de manera directa de la memoria semántica del sujeto, y el orden otorgado va de acuerdo con su escala de valores y percepciones. Cuando se le pide al sujeto que mencione las palabras que definen al concepto (palabra estímulo), este hurga en su memoria y selecciona aquellas que asume más relacionadas; en este sentido, la elección es resultado de un proceso subjetivo de representarse al mundo (en particular a la palabra estímulo). Así, la mediación del investigador se limita a la estimulación; no interviene durante la búsqueda y la selección de las palabras con las que el sujeto define al objeto. Esto le da a la técnica un carácter "natural” y "abierto", mientras que lo "cualitativo" se genera durante el proceso de registro, porque 


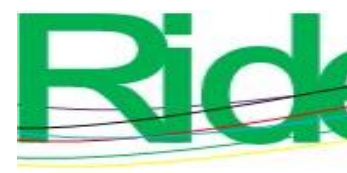

Revista Iberoamericana para la
Investigación y el Desarrollo Educativo
ISSN $2007-7467$

lo que obtenemos son palabras en lenguaje natural (Zermeño, Arellano y Ramírez, 2005, p. 310).

Asimismo, Zermeño et al. (2005) mencionan que "el conocimiento sobre el mundo se construye en forma de red y de manera jerárquica” (311). Figueroa, González y Solís (citados en Zermeño et al., 2005) entienden, por su parte, que una alternativa para evaluar el significado es la técnica de redes semánticas porque la forma interna para organizar la información en la memoria a corto plazo es en una red que vincula a las palabras y a los eventos, lo que genera el significado de un concepto.

De tal forma que, cuando el sujeto se ve en la necesidad de nombrar o significar la realidad, reconstruye la información al "jalar” de las relaciones para obtener los significados de los conceptos (nodos); en definitiva, este proceso es el conocimiento, y puede materializarse con las redes semánticas naturales (Zermeño et al., 2005, p. 312).

La asociación de palabras consistió en establecer una palabra o frase inductora, en este caso docencia universitaria y prácticas pedagógicas del docente universitario, y se solicitó a los estudiantes participantes de las diferentes carreras que conforman el colegio de ciencias de la salud de una universidad pública que escribieran cinco palabras que asociaran espontáneamente con esos términos, para después jerarquizarlas en orden de importancia, en donde 1 es el más importante y 5 el menos importante.

\section{Caracterización de los sujetos}

Los sujetos de estudio cursan cualquier semestre y cualquier licenciatura del colegio de ciencias de la salud de una universidad pública de la ciudad de Culiacán, Sinaloa, México. Por cada licenciatura se aplicaron 50 redes semánticas naturales para determinar las representaciones sociales de la docencia universitaria y 50 redes semánticas naturales para determinar las representaciones sociales de las prácticas pedagógicas de los docentes universitarios; en cada uno de los casos, las encuestas fueron contestadas por 25 hombres y 25 mujeres, cuya edad oscilaba entre los 18 y 22 años. En total, se aplicaron 1200 redes semánticas naturales (ver tabla 1). Los estudiantes seleccionados tuvieron disposición de contestar y estructurar la red semántica después de firmar la carta de consentimiento informado. 
Tabla 1. Distribución de programas educativos del colegio de ciencias de la salud y número de estudiantes a quienes se les aplicó el instrumento de redes semánticas de representaciones sociales

\begin{tabular}{|l|c|c|}
\hline \multicolumn{1}{|c|}{ Programa educativo } & Estudiantes & Total \\
\hline Lic. en Enfermería & 100 & 100 \\
\hline Lic. en Medicina & 100 & 100 \\
\hline Lic. en Fisioterapia & 100 & 100 \\
\hline Lic. en Gericultura & 100 & 100 \\
\hline Lic. en Cirujano Dentista & 100 & 100 \\
\hline Lic. en Psicología & 100 & 100 \\
\hline Lic. en Nutrición & 100 & 100 \\
\hline $\begin{array}{l}\text { Lic. en Químico } \\
\text { Farmacéutico Biólogo }\end{array}$ & 100 & 100 \\
\hline Lic. en Actividad Física & & $\mathbf{6 0 0}$ \\
para la Salud & 100 & 100 \\
\hline $\begin{array}{l}\text { Lic. en Educación Física y } \\
\text { Ciencias del Deporte }\end{array}$ & 100 & 100 \\
\hline Lic. en Optometría & 100 & \\
\hline Lic. en Biomedicina & & \\
\hline Total & 100 & \\
\hline
\end{tabular}

Fuente: Elaboración propia

\section{Resultados}

Se determinó el valor J, que es el conteo final de las palabras definidoras diferentes. El resultado indica la riqueza semántica de la red. Luego, se hicieron las operaciones para obtener el valor M, que indica el peso semántico y se obtiene al multiplicar individualmente la frecuencia de aparición (Fa) de cada palabra definidora por el valor semántico (VS) que se otorga de acuerdo con la jerarquía asignada; al final se suman los valores resultantes (Valdez, 1998) (ver tabla 2 y 3). 


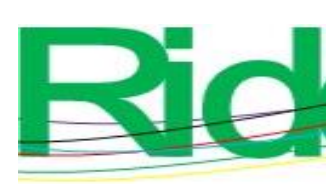

e

Revista Iberoamericana para la Investigación y el Desarrollo Educativo ISSN 2007 - 7467

Tabla 2. Obtención del valor $M$ de las representaciones sociales de la docencia universitaria

\begin{tabular}{|c|c|c|c|c|c|c|c|c|c|c|c|}
\hline $\begin{array}{c}\text { Jerarquía } \\
\text { asignada }\end{array}$ & 1 & 2 & 3 & 4 & 5 & \multirow{2}{*}{\multicolumn{5}{|c|}{$\begin{array}{c}\text { Frecuencia de aparición } \\
\qquad \mathbf{x} \\
\text { Valor semántico }\end{array}$}} & \multirow{3}{*}{$\begin{array}{c}\text { Suma } \\
= \\
\text { Valor M }\end{array}$} \\
\hline & 5 & 4 & 3 & 2 & 1 & & & & & & \\
\hline Definidoras & $\mathrm{Fa}$ & $\mathrm{Fa}$ & $\mathrm{Fa}$ & $\mathrm{Fa}$ & $\mathrm{Fa}$ & FaX5 & FaX4 & FaX3 & $\mathrm{FaX} 2$ & FaX1 & \\
\hline Preparado & $\begin{array}{l}23 \\
5\end{array}$ & $\begin{array}{l}11 \\
4\end{array}$ & 95 & 57 & 22 & 1175 & 456 & 285 & 114 & 22 & 2052 \\
\hline Confianza & $\begin{array}{l}16 \\
6\end{array}$ & $\begin{array}{l}11 \\
1\end{array}$ & 87 & 34 & 27 & 830 & 444 & 261 & 68 & 27 & 1630 \\
\hline Responsable & $\begin{array}{l}18 \\
4\end{array}$ & $\begin{array}{l}11 \\
7\end{array}$ & 49 & 12 & 09 & 920 & 468 & 147 & 24 & 09 & 1568 \\
\hline $\begin{array}{l}\text { Comprometid } \\
\text { o }\end{array}$ & $\begin{array}{l}15 \\
4\end{array}$ & $\begin{array}{l}11 \\
3\end{array}$ & 39 & 32 & 27 & 770 & 452 & 117 & 64 & 27 & 1430 \\
\hline Asertivo & 98 & 79 & 49 & 16 & 15 & 490 & 316 & 147 & 32 & 15 & 1000 \\
\hline Tutor & 90 & 78 & 50 & 12 & 11 & 450 & 312 & 150 & 24 & 11 & 947 \\
\hline Asesor & 88 & 72 & 48 & 15 & 12 & 440 & 288 & 144 & 30 & 11 & 913 \\
\hline $\begin{array}{c}\text { Desactualiza } \\
\text { do }\end{array}$ & 79 & 67 & 50 & 11 & 10 & 395 & 268 & 150 & 22 & 10 & 845 \\
\hline Tradicional & 75 & 70 & 38 & 18 & 05 & 375 & 280 & 114 & 36 & 05 & 810 \\
\hline $\begin{array}{c}\text { Sin } \\
\text { preparación }\end{array}$ & 64 & 62 & 28 & 11 & 10 & 320 & 248 & 84 & 22 & 10 & 684 \\
\hline
\end{tabular}

Fuente: Elaboración propia 


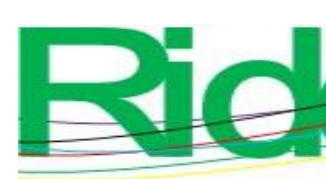

Revista Iberoamericana para la Investigación y el Desarrollo Educativo ISSN 2007 - 7467

Tabla 3. Obtención del valor $\mathrm{M}$ de las representaciones sociales sobre las prácticas pedagógicas de los docentes universitarios

\begin{tabular}{|c|c|c|c|c|c|c|c|c|c|c|c|}
\hline $\begin{array}{c}\text { Jerarquía } \\
\text { asignada }\end{array}$ & 1 & 2 & 3 & 4 & 5 & \multirow{2}{*}{\multicolumn{5}{|c|}{$\begin{array}{c}\text { Frecuencia de aparición } \\
\qquad x \\
\text { Valor semántico }\end{array}$}} & \multirow{3}{*}{$\begin{array}{c}\text { Suma } \\
\quad= \\
\text { Valor M }\end{array}$} \\
\hline $\begin{array}{c}\text { Valor } \\
\text { semántico }\end{array}$ & 5 & 4 & 3 & 2 & 1 & & & & & & \\
\hline Definidoras & $\mathrm{Fa}$ & $\mathrm{Fa}$ & $\mathrm{Fa}$ & $\mathrm{Fa}$ & $\mathrm{Fa}$ & FaX5 & FaX4 & FaX3 & FaX2 & FaX1 & \\
\hline Examen & $\begin{array}{l}29 \\
4\end{array}$ & $\begin{array}{l}11 \\
8\end{array}$ & 88 & 45 & 44 & 1470 & 472 & 264 & 90 & 44 & 2340 \\
\hline Exposición & $\begin{array}{l}25 \\
6\end{array}$ & 98 & 36 & 32 & 22 & 1280 & 392 & 108 & 64 & 22 & 1866 \\
\hline $\begin{array}{c}\text { Mapas } \\
\text { conceptuales }\end{array}$ & $\begin{array}{l}18 \\
4\end{array}$ & 60 & 52 & 22 & 07 & 920 & 240 & 156 & 44 & 07 & 1367 \\
\hline Resumen & 91 & 88 & 44 & 29 & 13 & 455 & 352 & 132 & 58 & 13 & 1010 \\
\hline $\begin{array}{l}\text { Cuadro } \\
\text { sinóptico }\end{array}$ & 72 & 52 & 28 & 17 & 04 & 360 & 208 & 84 & 34 & 04 & 690 \\
\hline Caso clínico & 71 & 50 & 27 & 15 & 10 & 355 & 200 & 81 & 30 & 10 & 676 \\
\hline Debate & 70 & 51 & 25 & 15 & 08 & 350 & 204 & 75 & 30 & 08 & 667 \\
\hline $\begin{array}{c}\text { Simulación } \\
\text { clínica }\end{array}$ & 63 & 50 & 30 & 10 & 08 & 315 & 200 & 90 & 20 & 08 & 633 \\
\hline Sociodrama & 60 & 32 & 27 & 18 & 05 & 300 & 128 & 81 & 36 & 05 & 550 \\
\hline Ensayo & 38 & 30 & 16 & 12 & 08 & 190 & 120 & 48 & 24 & 08 & 390 \\
\hline
\end{tabular}

Fuente: Elaboración propia

Con esto, se identificó el conjunto SAM, que es el conjunto de las 10 definidoras que obtienen el mayor peso semántico y que son el núcleo central de la red. También se obtuvo el valor FMG (ver tabla 4 y 5), que permite observar la distancia semántica entre las palabras de la red, es decir, indica la cercanía de las palabras con respecto al término inductor. Para determinarlo, se otorga $100 \%$ al valor más alto y con una regla de tres se determinan los valores subsiguientes. Esta operación revela datos de la jerarquización de los elementos del núcleo central y aporta información para entender el significado (Valdez, 1998). 


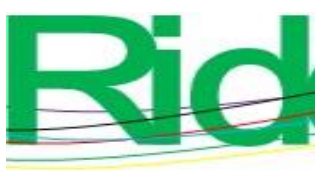

Revista Iberoamericana para la Investigación y el Desarrollo Educativo ISSN $2007-7467$

Tabla 4. Núcleo central de la red, peso y distancia del sistema periférico de las representaciones sociales de la docencia universitaria

\begin{tabular}{|l|c|c|}
\hline \multicolumn{1}{|c|}{ Núcleo central de la red } & Peso semántico & Distancia semántica \\
\hline 1) Preparado & 2052 & 100 \\
\hline 2) Confianza & 1630 & 79.43 \\
\hline 3) Responsable & 1568 & 76.41 \\
\hline 4) Comprometido & 1433 & 69.83 \\
\hline 5) Asertivo & 1000 & 48.73 \\
\hline 6) Tutor & 947 & 46.15 \\
\hline 7) Asesor & 913 & 44.49 \\
\hline 8) Desactualizado & 845 & 41.17 \\
\hline 9) Tradicional & 810 & 39.47 \\
\hline 10) Sin Pedagogía & 684 & 33.33 \\
\hline
\end{tabular}

Fuente: Elaboración propia

Tabla 5. Núcleo central de la red, peso y distancia del sistema periférico de las representaciones sociales sobre las prácticas pedagógicas de los docentes universitarios

\begin{tabular}{|l|c|c|}
\hline \multicolumn{1}{|c|}{ Núcleo central de la red } & Peso semántico & Distancia semántica \\
\hline 1) Examen & 2340 & 100 \\
\hline 2) Exposición & 1866 & 79.74 \\
\hline 3) Mapas conceptuales & 1367 & 58.41 \\
\hline 4) Resumen & 1010 & 43.16 \\
\hline 5) Cuadro sinóptico & 690 & 29.48 \\
\hline 6) Caso clínico & 676 & 28.88 \\
\hline 7) Debate & 667 & 28.50 \\
\hline 8) Simulación clínica & 633 & 27.05 \\
\hline 9) Sociodrama & 550 & 23.50 \\
\hline 10) Ensayo & 390 & 16.66 \\
\hline
\end{tabular}

Fuente: Elaboración propia 

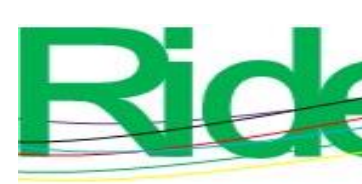

Figura 1. Núcleo central de la red, peso y distancia del sistema periférico de las representaciones sociales de la docencia universitaria

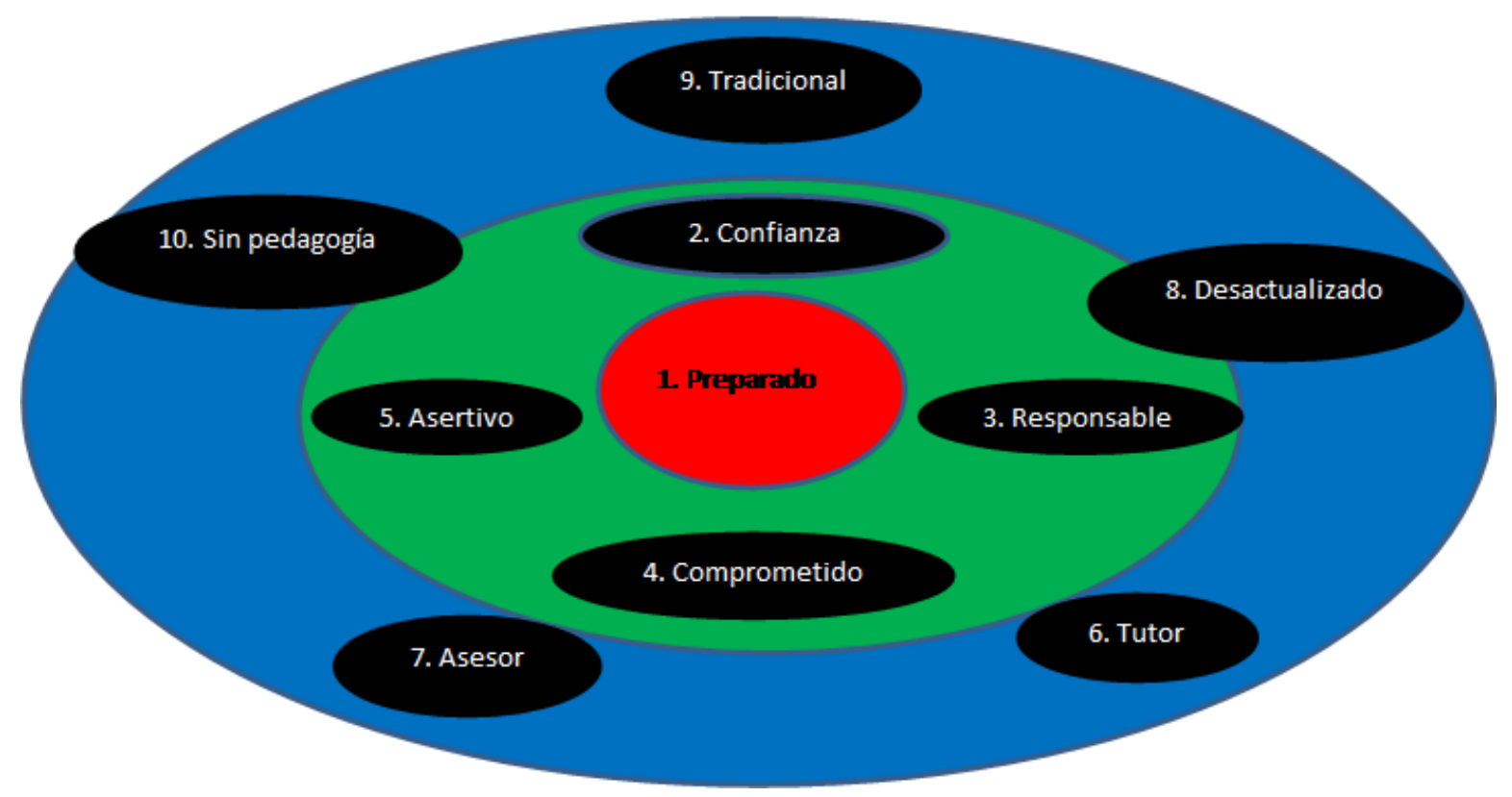

Fuente: Elaboración propia

De acuerdo con las redes de asociaciones que se presentan en la configuración del núcleo central de las representaciones sociales de la figura 1, se determinó que el núcleo figurativo de "Docencia universitaria" recae en el concepto central de preparado, es decir, estar preparado para impartir la clase, seguido de los conceptos que integran el sistema periférico de la representaciones sociales: confianza, responsable, comprometido y asertivo. Todos estos componentes son básicos para determinar las redes de asociaciones.

El núcleo central es el elemento que más resistirá al cambio, pues una modificación del núcleo produce la transformación completa de la representación. Está protegido, por tanto, por los sistemas periféricos, los cuales permiten, esencialmente, la adaptación de la representación a las evoluciones del contexto. Los elementos periféricos están en relación directa con el núcleo, lo cual equivale a decir que su presencia, su ponderación, su valor y su función están determinados por el núcleo. Están jerarquizados: pueden estar muy cerca de los elementos centrales y en este caso juegan un importante papel en la concreción del significado de la representación (Abric, 1994). 

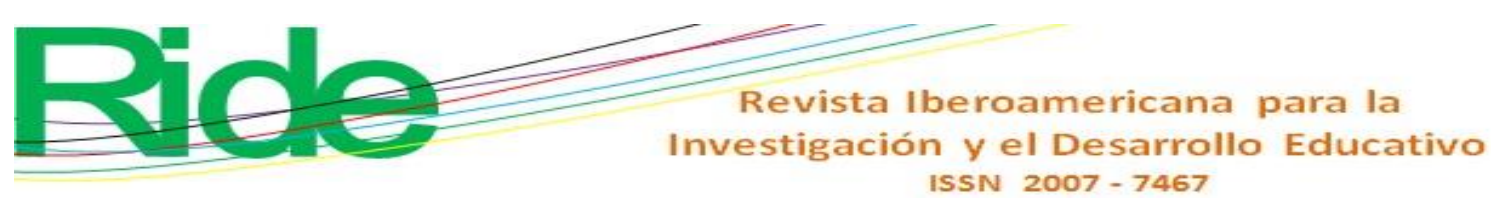

Una de las mayores virtudes de trabajar con las redes semánticas es que muestran la organización y jerarquía de las palabras evocadas, pues no sería útil quedarse con asociaciones de palabras como conglomerados azarosos o listas sin sentido. Para observar el contenido de la representación social, se requiere observar las relaciones de las palabras, ver cómo están concatenadas, organizadas y, gracias a ello, develar parte del sentido común de los actores educativos en torno al objeto representado (Mireles, 2014, p. 8).

Figura 2: Núcleo central de la red, peso y distancia del sistema periférico de las representaciones sociales sobre las prácticas pedagógicas de los docentes universitarios

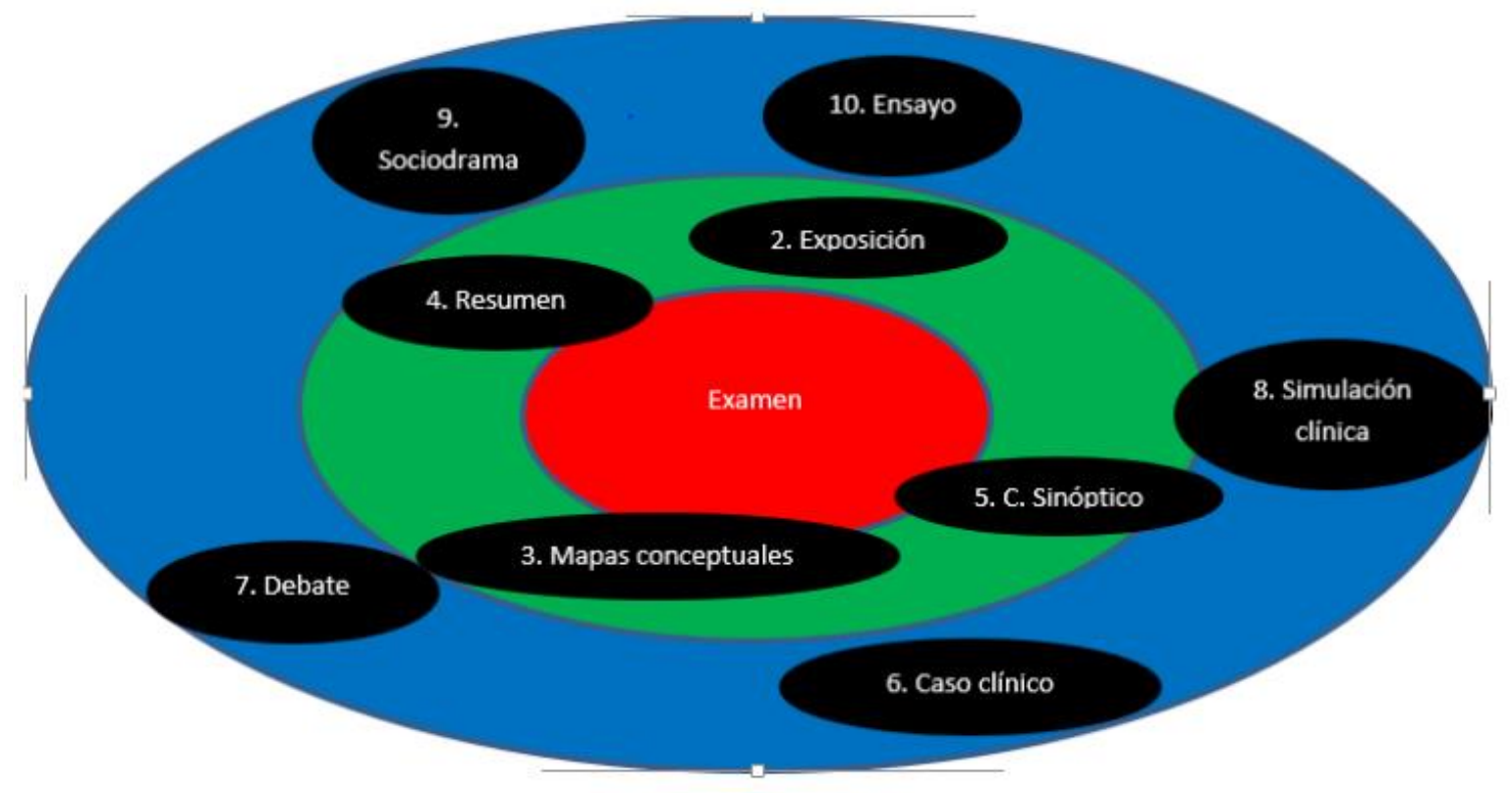

Fuente: Elaboración propia

Ahora bien, siguiendo las redes de asociaciones que se presentan en la configuración del núcleo central de las representaciones sociales patentes en la figura 2, se determinó que el núcleo figurativo de "Prácticas pedagógicas" recae en el concepto central de examen, esto es, el examen como herramienta principal para evaluar los aprendizajes de los estudiantes del área de la salud, seguido de los conceptos que integran el sistema periférico de la representaciones sociales: exposición, cuadro sinóptico, mapas conceptuales y resumen. Todos estos componentes son básicos para determinar las redes de asociaciones. 


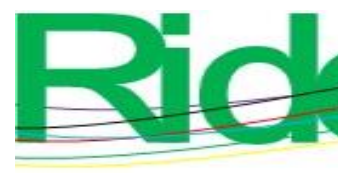

Revista Iberoamericana para la
Investigación y el Desarrollo Educativo
ISSN $2007-7467$

Según Abric (1994), las representaciones sociales cumplen con tres funciones:

- Función de concreción: directamente dependientes del contexto, resultan del anclaje de la representación en la realidad permitiendo su investidura en términos concretos, comprensibles y transmisibles de inmediato. Integran los elementos de la situación en la que la representación se produce, refieren el presente y lo vivido por las personas (Abric, 1994).

- Función de regulación: por su mayor flexibilidad en relación con los elementos centrales, los elementos periféricos desempeñan un papel esencial en la adaptación de la representación a la evolución del contexto. De esta forma, cualquier información nueva o transformación del entorno se integra a la periferia. Frente a la estabilidad del núcleo central, constituyen el aspecto móvil y evolutivo de la representación (Abric, 1994).

- Función de defensa: el sistema periférico cumple una función de "parachoques" al proteger al núcleo central de su eventual transformación. Si el núcleo central cambia es porque el sistema periférico es poco resistente o porque las nuevas informaciones contienen mucha fuerza. En todo caso, es el sistema periférico el que soporta las primeras transformaciones: cambios de ponderación, interpretaciones nuevas, deformaciones funcionales defensivas, integración de condicional de elementos contradictorios (Abric, 1994).

\section{Discusión}

En la investigación de Banegas y Fornasero (2004) se encontró que el docente es "el actor central que dirige, guía, media, facilita el proceso educativo" (p. 171); por su parte, en dicho proceso el alumno tiene una función activa.

El docente "es aquella persona que se ha formado (su desafío actual es la capacitación, la formación continua y la actualización) en una disciplina y está investido de responsabilidad institucional para enseñar”. Enseñar, a su vez, es transferir conocimientos, valores, ideas, etc. con el "propósito de favorecer la construcción de conocimientos" en los alumnos. El alumno es el "protagonista de su propio proceso educativo", a quien "deberían respetarse, especialmente, sus potencialidades psicológicas, intereses, actitudes para orientar la enseñanza y el aprendizaje". Aprender es sinónimo de "cambio, de modificación, de incorporación valorativa" de los conocimientos (Benegas y Fornasero, 2004, p. 171). 


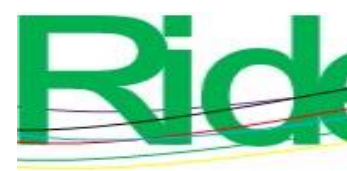

Revista Iberoamericana para la
Investigación y el Desarrollo Educativo
ISSN $2007-7467$

En nuestra investigación se encontró que las principales redes de asociaciones de representaciones sociales sobre la docencia universitaria se determinaron a partir del peso semántico de cada uno de los conceptos o categorías centrales: preparado con un peso semántico de 100 , confianza con un peso semántico de 79.43 , responsable con un peso semántico de 76.41, comprometido con un peso semántico de 69.83 y asertivo con un peso semántico de 48.73 .

La representación no es un simple reflejo de la realidad sino una organización significante (...). Funciona como un sistema de interpretación de la realidad que rige las relaciones de los individuos con su entorno físico y social, ya que determinará sus comportamientos o sus prácticas. Es una guía para la acción, orienta las acciones y las relaciones sociales. Es un sistema de pre-decodificación de la realidad puesto que determina un conjunto de anticipaciones y expectativas (Abric, 2001, p. 17).

Por otro lado, Arbesú y Hernández (2015) encontraron que los estudiantes valoran que los docentes dominen diversas estrategias de enseñanza, de tal forma que la clase sea dinámica, atractiva y esto tenga como resultado una mayor motivación por aprender por parte del alumno.

Asimismo, entre las estrategias que privilegian los alumnos, destacan aquellas que permiten establecer un vínculo entre la teoría y la práctica, es decir, que consideran que aprenden con mayor facilidad cuando el profesor les ayuda a entender de qué forma los contenidos aprendidos pueden serles útiles en situaciones reales (Arbesú y Hernández, 2015, p. 53).

En nuestra investigación, se encontró que las principales redes de asociaciones de representaciones sociales sobre las prácticas pedagógicas de los docentes universitarios se determinaron a partir del peso semántico de cada uno de los conceptos o categorías centrales: examen con un peso semántico de 100, exposición con peso semántico de 79.74 , mapas conceptuales con un peso semántico de 58.41, resumen con un peso semántico de 43.16 y cuadro sinóptico con un peso semántico de 29.48 .

Las representaciones sociales como forma de conocimiento aluden a un proceso y a un contenido. En tanto proceso, estas refieren a una forma particular de adquirir y comunicar conocimientos (Moscovici, 1979). 

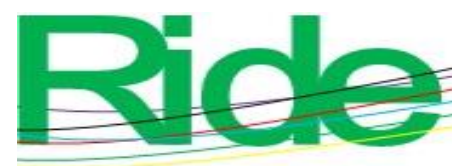

Revista Iberoamericana para la Investigación y el Desarrollo Educativo ISSN 2007 - 7467

\section{Conclusiones}

Las redes de asociaciones de las respuestas de los estudiantes universitarios de ciencias de la salud que se presentan en la configuración del núcleo central de las representaciones sociales de la docencia universitaria, que corresponden a la primera red de asociación, permiten determinar el contenido y organización de la representación social. Se encuentra principalmente en el concepto de preparado, es decir, que los estudiantes reconocen que sus docentes conocen las unidades de aprendizaje que se les asignan en las diferentes profesiones que componen el área de la salud.

En lo que corresponde a la segunda red de asociación, referente a las prácticas pedagógicas de la docencia universitaria, en este caso el núcleo figurativo se determinó en el concepto de examen. A pesar de que los docentes universitarios del área de la salud cuentan con diferentes herramientas para evaluar los aprendizajes de sus estudiantes, se sigue utilizando el examen como principal herramienta de evaluación.

Estos hallazgos permiten establecer nuevas líneas de investigación aplicando la teoría de las representaciones sociales en diferentes objetos de estudio del área de la educación (modelo educativo, evaluación, calidad de la educación superior, diversificación de la oferta educativa, trayectorias estudiantiles). 


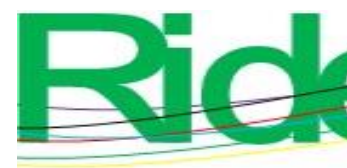

Revista Iberoamericana para la
Investigación y el Desarrollo Educativo ISSN $2007-7467$

\section{Referencias}

Abric, J. (1994). Metodología de recolección de las representaciones sociales. Ciudad de México, México: Ediciones Coyoacán.

Abric, J. (2001). Prácticas sociales y representaciones. Ciudad de México, México: Ediciones Coyoacán.

Amber, D. A. y Suárez, C. I. (2016). La formación docente universitaria: claves formativas de universidades españolas. Revista Electrónica de Investigación Educativa, 18(3), 51-64. Recuperado de http://redie.uabc.mx/redie/article/view/996.

Araya, S. (2002). Las representaciones sociales: Ejes teóricos para su discusión. San José, Costa Rica: Facultad Latinoamericana de Ciencias Sociales (FLACSO).

Arbesú, M. I. y Reyes, L. (2015). La eficacia docente: Representaciones sociales de estudiantes universitarios. Observar. Revista Electrónica de Didáctica de Las Artes, (9), 37-57. Recuperado de https://www.observar.eu/index.php/Observar/article/view/62.

Barbachán, E., Cajas, T., Ramos, F. y Sánchez, F. (2017). Representaciones sociales de la seguridad ciudadana en estudiantes universitarios de Lima-Perú. Opción, 33(84), 698-724.

Beltrán, M. y Garay, J. (2016). Representaciones sociales de los métodos anticonceptivos. Revista Iberoamericana de las Ciencias de la Salud, 5(10).

Benegas, M. A. y Fornasero, S. (2004). Representaciones sociales y modelos pedagógicos de alumnos y docentes universitarios. Educación, Lenguaje y Sociedad, 2(2). Recuperado http://www.biblioteca.unlpam.edu.ar/pubpdf/ieles/n02a07benegas.pdf.

Berger, P. y Luckmann, T. (1991). La construcción social de la realidad. Buenos Aires, Argentina: Amorrortu editores.

Covarrubias, P. P. (2011). Representaciones sobre la enseñanza. Una indagación en estudiantes universitarios. Sinéctica (36), 1-18. Recuperado de http://www.scielo.org.mx/scielo.php?script=sci_arttext\&pid=S1665109X2011000 10002.

Covarrubias, P. P. (2016). Representaciones docentes de la Educación Basada en Competencias. Un estudio de caso. Propósitos y Representaciones, 4(2), 73-132. Recuperado de http://revistas.usil.edu.pe/index.php/pyr/article/view/120/221. 


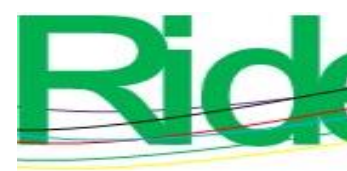

Revista Iberoamericana para la
Investigación y el Desarrollo Educativo
ISSN $2007-7467$

Figueroa, J. G., González, E. y Solís, V. (1981). Una aproximación al problema del significado: las redes semántica. Revista Latinoamericana de Psicología, 13(3), 447-458.

Recuperado

de http://catalogosuba.sisbi.uba.ar/vufind/Record/201603170442033945/Details.

Hernández, G. (2006). Paradigmas en psicología de la educación. México: Paidós.

Ibáñez, T. (1988). Ideologías de la vida cotidiana. Barcelona, España: Sendai.

Jodelet, D. (1986). La representación social: fenómeno, concepto y teoría. En Moscovici, S. (ed.), Psicología social II. España: Paidós.

Jodelet, D. (2006). Place de l'expérience vécue dans les processus de formation des représentations sociales. En Haas, V. (dir.), Les savoirs du quotidien. France: Presses universitaires de Rennes.

Mireles, O. (2014). Asociación de palabras y redes semánticas: recursos metodológicos para develar representaciones sociales. Un caso de investigación educativa. Ponencia presentada en la 3. ${ }^{\text {a }}$ Conferencia Internacional Multidisciplinaria de Investigación Educativa. Segovia, del 3 al 4 de julio de 2014. Recuperado de http://amieedu.org/actascimie14/wp-content/uploads/2014/12/Mireles-A7Asociaci\%C3\%B3n-y-redes.pdf.

Moscovici, S. (1979). El psicoanálisis, su imagen y su público. Buenos Aires, Argentina: Huemul.

Rueda, M. y Luna, E. (2008). Introducción: La docencia universitaria y su evaluación. REDIE. Revista Electrónica de Investigación Educativa, 10(número especial). Recuperado de https://www.redalyc.org/articulo.oa?id=155/15511127001.

Schwartz, H. y Jacobs, J. (1984). Sociología cualitativa. México: Trillas.

Tourón, J. (9 de marzo de 2015). ¿Cómo es el estudiante del siglo XXI? Javier Tourón. Porque el talento que no se cultiva, se pierde. Recuperado de https://www.javiertouron.es/como-es-el-estudiante-del-siglo-xxi/.

Universidad Autónoma de Sinaloa [UAS]. (2017). Modelo Educativo de la Universidad Autónoma de Sinaloa. México: Universidad Autónoma de Sinaloa. Recuperado de http://sau.uas.edu.mx/pdf/Modelo_Educativo_UAS_2017.pdf.

Valdez, J. L. (1998). Las redes semánticas naturales, uso y aplicaciones en psicología social. México: Universidad Autónoma del Estado de México. 

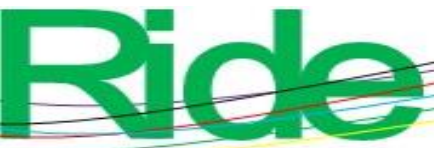

Revista Iberoamericana para la Investigación y el Desarrollo Educativo ISSN $2007-7467$

Zermeño, A., Arellano, A. y Ramírez, V. (2005). Redes semánticas naturales: técnica para representar los significados que los jóvenes tienen sobre televisión, Internet y expectativas de vida. Estudios sobre las Culturas Contemporáneas, 11(22), 305334. Recuperado de redalyc.org/pdf/316/31602207.pdf.

Zúñiga, M. (2012). Los estudiantes universitarios del siglo XXI en México: de la pasividad a la autonomía y al pensamiento crítico. Revista Teoría de la Educación: Educación y Cultura en la Sociedad de la Información, 13(2), 424-440. Recuperado de http://campus.usal.es/ revistas_trabajo/index.php/revistatesi/article/view/9021/92 65. 